\title{
Methanol masers: evolutionary and kinematic tracers of massive star formation
}

\author{
Vincent Minier, Roy Booth, John Conway, and Michele Pestalozzi \\ Onsala Space Observatory, 439 92 Onsala, Sweden
}

\begin{abstract}
We summarise our recent VLBI observations of a large sample of methanol maser sources associated with high-mass star-forming regions.
\end{abstract}

\section{Introduction}

Since the discoveries of the strongest methanol masers at 6.7 and $12.2 \mathrm{GHz}$ ten years ago, the field of methanol maser research has undergone rapid development. We have recently conducted at the Onsala Space Observatory an extensive study of the methanol masers in the Northern hemisphere. 6.7 and $12.2 \mathrm{GHz}$ methanol masers have been mapped in 15 massive star-forming regions using the European VLBI Network (EVN) and the VLBA (Minier et al. 2000). In parallel, a blind survey of the Galactic plane has been undertaken in order to discover new 6.7 $\mathrm{GHz}$ methanol maser sources using the Onsala-25m antenna, as well as followup observations of these new sources using molecular lines in the range 85$112 \mathrm{GHz}$ (see Pestalozzi et al. this volume). Although these latest observations are generally consistent with previous work done in Australia, new conclusions have been reached, making the research in methanol masers even more exciting and convincing to understand the process of massive (proto)stellar evolution still mainly unknown. We summarise in this paper the VLBI results.

\section{Methanol masers: evolutionary tracers}

Most of the reported class II methanol masers are associated with the traditional signposts of massive star formation, i.e luminous infrared sources, UC HII regions, bipolar outflows, $\mathrm{OH}$ and $\mathrm{H}_{2} \mathrm{O}$ masers. However, when the methanol masers appear to be associated with UC HII regions from single dish observations, high resolution imaging shows that the majority of maser sites are not superimposed on the UC HII regions (Minier et al. 2001a; Walsh et al. 1998). Instead they lie clearly offset in position from the ionised region and could mark the location of another young stellar object. Comparisons with images of the star-forming regions at other wavelengths suggest that the methanol maser sites coincide in position with hot molecular cores (Fig. 1), outflows and hyper compact HII regions, making the methanol masers potential tools to understand the protostellar evolution of massive stars (see Minier et al. 2001a). In consequence, assuming that $\mathrm{CH}_{3} \mathrm{OH}$ masers only form in the environment of massive stellar objects, they are perhaps one of the first signposts of massive protostars prior to 


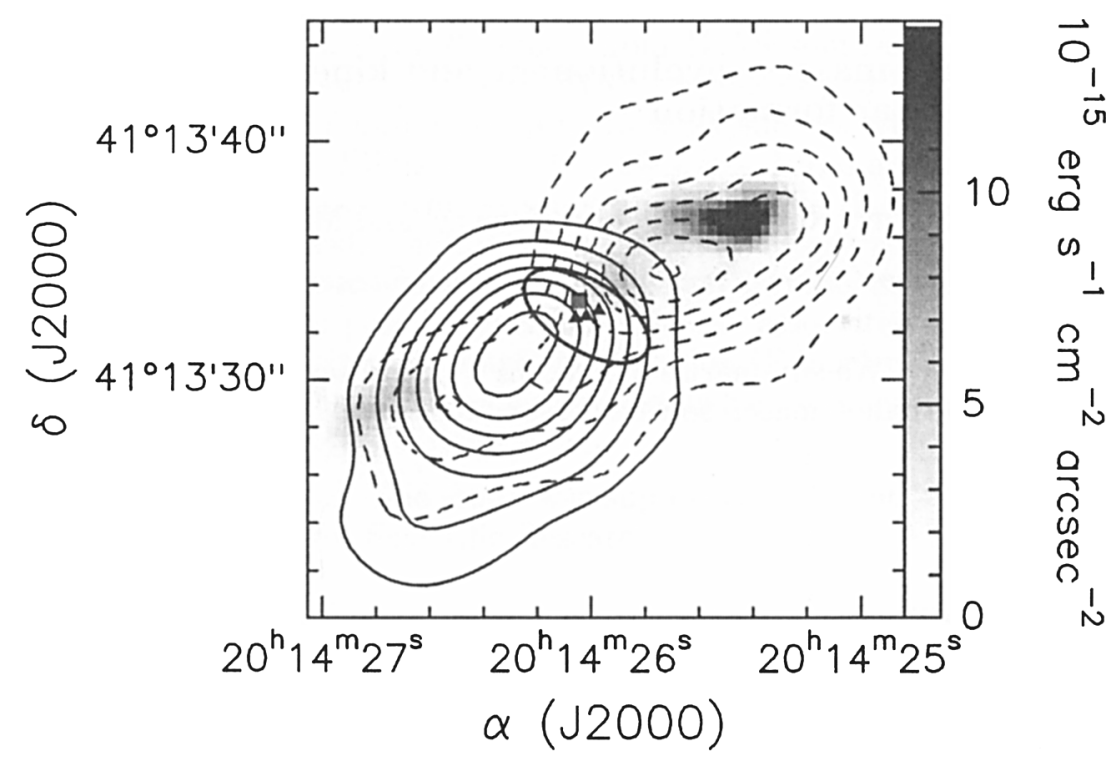

Figure 1. The massive protostar IRAS 20126+4104: contours represent the blue (continuous line) and redshifted (dashed line) components of the $\mathrm{HCO}^{+}$outflow. The grey scale represents the $\mathrm{H}_{2}$ emission. The $\mathrm{H}_{2} \mathrm{O}$ (triangles) and $\mathrm{CH}_{3} \mathrm{OH}$ (square) masers are seen projected onto the $\mathrm{NH}_{3}$ disk (ellipse)

the development of UC HII regions. Then, they evolve with the protostar and are progressively destroyed during the UC HII phase as argued by Walsh et al. (1998).

\section{Methanol masers: kinematic tracers}

Our VLBI observations toward 15 star-forming regions show that the methanol masers possibly trace either (proto)stellar disks in Keplerian motion around a high central mass or shocked clumps within outflows. In many sources the masers trace disklike structures of only a few tens to a few hundreds of $\mathrm{AU}$ and the derived central masses assuming Keplerian motion are less than $1 \mathrm{M}_{\odot}$. One possible explanation is that the methanol masers partially trace a disk, and hence the derived mass is a lower limit of the central star mass (Fig. 2). Some evidence for methanol masers tracing outflows is also found in $\mathrm{W} 75 \mathrm{~N}$, NGC 7538 (Fig. 2) and G 9.62+0.20 (Minier et al. 2001b).

\section{The milliarcsecond structures of methanol masers}

After dissecting with the VLBI the intrinsic maser structures of three representative methanol maser sites, we argue that the majority of the individual masing regions within a maser site consist of a compact core surrounded by extended 

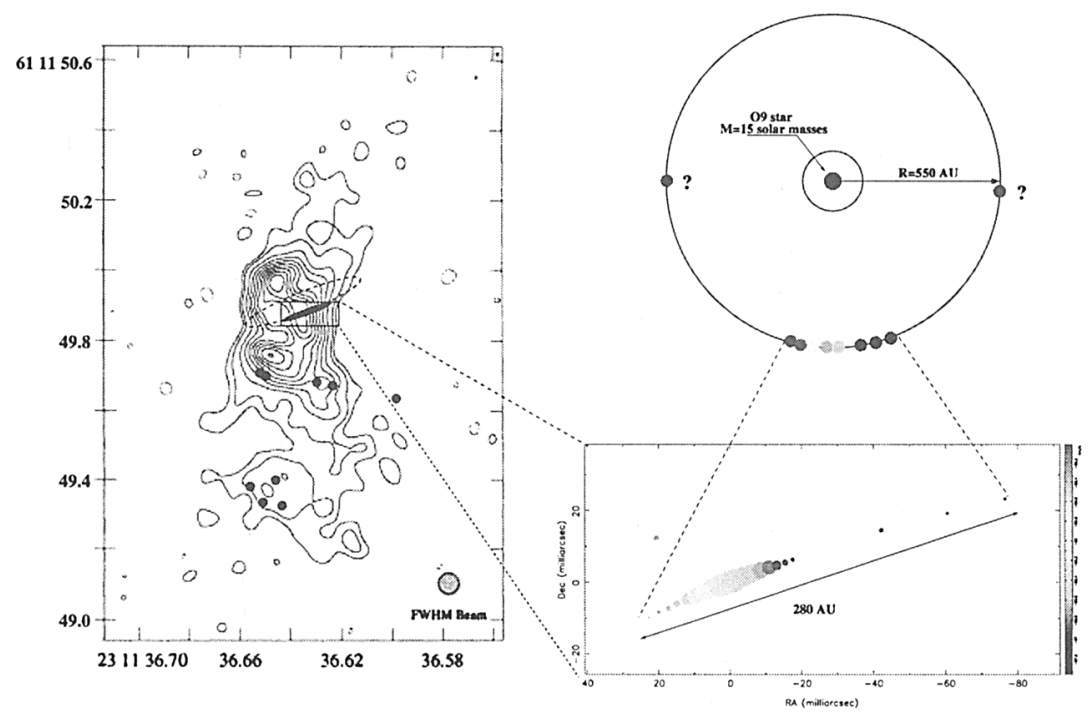

Figure 2. Left: $6.7 \mathrm{GHz}$ methanol maser distribution (black symbol) in NGC 7538-IRS1 overlaid on the $22 \mathrm{GHz}$ radio continuum emission (contour) that trace a north-south ionised outflow. Masers are associated with clumps in the radio emission and with a linear structure. Right: Masers partially tracing an edge-on circumstellar disk in NGC 7538-IRS1. The grey scale indicates the velocity. The dot size is proportional to the intensity of the maser component. From radio continuum observations, the exciting star responsible for the central ionised region coincident with the maser site could be a 09.5 star, having a mass of $\sim 15 \mathrm{M}_{\odot}$. Using the linear velocity gradient along the maser line, the derived radius of the Keplerian disk is $550 \mathrm{AU}$ which is larger than the measured half-diameter of the line of masers. Masers are probably seen in front of the star, but no maser is detected on the edge of the disk

emission, i.e a core/halo structure (Minier et al. 2001 in prep.). An illustrative case is given in Fig. 3. The halos of diffuse emission have diameters ranging between 12 and $290 \mathrm{AU}$. The core diameters vary from 2 to $20 \mathrm{AU}$. Two possible models might explain the presence of large halos around bright cores: (1) the compact and diffuse masers could arise from two physical regions with different physical and chemical conditions; (2) the core/halo pattern could be the result of turbulence and saturation effects in a homogeneous region. The scattering effects do not play a role in the broadening of maser images.

\section{References}

Minier, V., Booth, R.S., \& Conway, J.E. 2000, A\&A, 362, 1093 Minier, V., Conway, J.E., \& Booth, R.S. 2001a, A\&A, 369, 278 Minier, V., Booth, R.S., Ellingsen, S.P. et al., 2001b, in Proceedings of the 5th EVN Symposium, ed. J.E. Conway, A.G. Polatidis, R.S. Booth, \& Y. Pihlstroem, Chalmers Technical University, Gothenburg, Sweden, p. 179 Walsh, A.J., Burton, M.G., Hyland, A.R., Robinson, G. 1998, MNRAS, 301, 640 
$69.62+0.20$ Nov 1998 (VLBA)

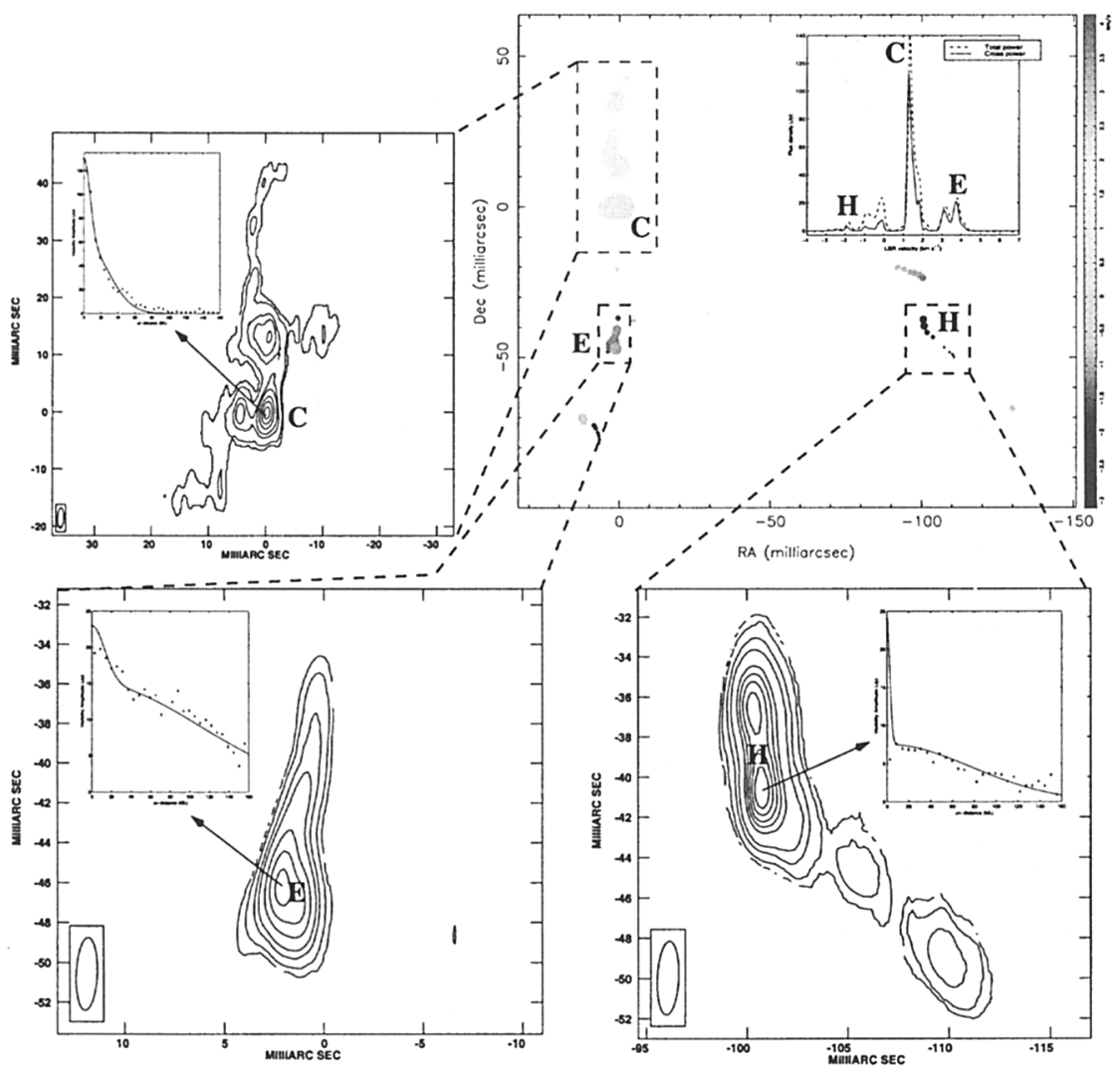

Figure 3. VLBI map of $12.2 \mathrm{GHz}$ methanol masers in G 9.62+0.20: the grey scale represents the velocity and the size of the maser dot is proportional to its intensity. The inset presents the cross-power spectrum on LA-PT and the total power spectrum on LA. It indicates that some flux is missing when comparing the cross-power spectrum with the single dish spectrum. Each close-up shows the integrated intensity over the velocity width for three spectral features $(\mathrm{C}, \mathrm{E}$, and $\mathrm{H})$. The inset in these images shows the visibility amplitude vs uv-distance at the peak in the contour image corresponding to the three peaks $\mathrm{C}, \mathrm{E}$ and $\mathrm{H}$ in the spectrum. The rapid decrease of the visibility with the baseline can be fitted with two Gaussian functions suggesting a double maser structure: a large halo of diffuse emission rapidly resolved and a compact core. We conclude that these regions of diffuse emission are responsible for the missing flux density when comparing the cross-power spectra on the shortest baselines to the single dish spectrum. We also conclude that methanol masers cannot only be represented as distinct spots 